\title{
Managing Emergent Character-Based Narrative
}

\author{
Ruth Aylett, Sandy Louchart \\ MACS \\ Heriot-Watt University \\ Edinburgh UK EH14 4AS \\ 0044(0)1314513424 \\ \{ruth, sandy\}@macs.hw.ac.uk
}

\author{
Anders Tychsen, Michael Hitchens \\ Department of Computing \\ Macquarie University \\ 2109 North Ryde, NSW, Australia \\ +0061 (02) 98509543 \\ \{atychsen,mhitchens\}@ics.mq.edu.au \\ Carlos Delgado Mata \\ Universidad Panamericana \\ Campus Bonaterra, Aguascalientes \\ Ags - C.P20290 - Mexico \\ cdelgado@ags.up.mx
}

\author{
Rui Figueiredo \\ INESC-ID and IST \\ Rua Prof Cavaco Silva \\ 2780990 Porto Salvo Portugal \\ Rui.figueiredo@tagus.ist.utl.pt
}

\begin{abstract}
In this paper, we consider the role of narrative management in a character-based emergent narrative framework. The paper defines the problem and considers related work. It evaluates the role of the Game Master in non computer-based role-playing games and presents two initial implementations of a story facilitator within a character-based system using the FAtiMA agent architecture. Finally it considers what further work is required.
\end{abstract}

\section{Categories and Subject Descriptors}

I.2.11 [Distributed Artificial Intelligence Language]: Intelligent agents.

\section{General Terms \\ Theory.}

\section{Keywords}

Synthetic actors, Artificial Intelligence, Interactive Storytelling. Story management. Game Masters

\section{Introduction}

Character-based or emergent narrative (EN) has been seen as a way of dealing with the conflict between the requirements of preauthored plot and user interactive freedom sometimes known as the narrative paradox [2] in interactive narrative. Here, the narrative experience consists of a dynamic process in which human users and synthetic characters jointly control and determine the unfolding of the narrative through their own autonomous choice of actions. However an argument against this approach is that just as a plot-directed approach may violate the behavioural consistency and thus the believability of characters, so autonomous characters may fail to produce any coherent, interesting narrative structure [20].

Permission to make digital or hard copies of all or part of this work for personal or classroom use is granted without fee provided that copies are not made or distributed for profit or commercial advantage and that copies bear this notice and the full citation on the first page. To copy otherwise, or republish, to post on servers or to redistribute to lists, requires prior specific permission and/or a fee. The Second International Conference on Intelligent Technologies for Interactive Entertainment (ICST INTETAIN '08). January 8-10, 2008, Cancun, Mexico. Copyright 2008 ICST. ISBN 978-963-9799$13-4$.
As part of a programme of work around the concept of emergent narrative, a number of solutions to this problem have been considered. For example, one may modify the architecture of synthetic characters such that their choice of autonomous action is influenced by dramatic requirements as well as their immediate goals [18]. In this paper however we argue that story facilitation, drawing on the functionality of the role-playing game (RPG) Game Master [9, 17, 32, 26, 27], is also worth investigating. The term story facilitation rather than management is used to emphasise the point that this is not a case of overruling the autonomous actions of either the user or the synthetic characters. It is a mechanism for shaping rather than for directing that is being investigated.

The problem of reconciling narrative structure with autonomous characters can actually be seen as a version of a very well-known problem in robotics. This is to accomplish tasks while retaining the ability to react to a changing environment. In both cases some higher-level structure is desired without removing the ability of agents to respond to what is happening around them. The classical view of planning as a one-to-many expansion of predetermined actions to be executed in the given sequence [11] corresponds to the plot-driven view of narrative. The purely character-based view of narrative has a certain analogy to behavioural robotics [6] in which all of an agent's actions are determined by the current world state.

In robotics, it came to be accepted that classical planning produced brittle and unresponsive systems [6]. However, purely behavioural systems could not offer any guarantee that desired outcomes would be reached and were plagued by problems associated with conflict resolution between competing behaviours and local minima [1]. Heterogeneous systems, combining planning with reactive execution in more sophisticated relationships than in the two extreme positions were seen as a solution [12]. Two such relationships are worthy of note in the context of interactive narrative.

The first of these was the use of planning as a resource by execution agents [33]. This requires agents to be able to detect local problems and refer to planning for global solutions. In interactive narrative, characters would need to be able to detect dramatic problems such as lack of narrative progression, absence of dramatic tension or possibly failure to reach specified states. A solution would then be requested from the story facilitator. In order to prevent such solutions conflicting with character 
consistency, negotiation would be required. This approach seems worth pursuing but is not the one adopted here.

A second approach sees planning as a context-setter for execution behaviours through the communication of constraints [1]. In robotics, where the real world is generally outside the control of the planning system, execution agents were given higher level information about which behaviours should be active in which contexts, so that for example obstacle avoidance could be turned off if a robot was trying to dock. In a virtual or story world however, the story facilitator has a great deal of control over the environment external to the characters. Mechanisms such as determining the outcome of physical actions, generation of exogenous events or the introduction of objects or opponents are well-used components of the Game Master's (GM's) repertoire in table-top role-playing. They are also used by the coordinator or facilitator of educational role-play. It is this approach we discuss below.

\section{Story-management in interactive systems}

Views of story-management are strongly determined by views of interactive narrative itself. It is worth bearing in mind two significant differences between the emergent narrative view adopted here and the views embodied in the related work considered.

An assumption of plot-based approaches is that the plot defines 'the' story. This does not imply that the story has to be exactly the same one each time the interactive system runs since this is clearly untrue of branching narrative approaches. However it does embody the concept of spectator, in that 'the' story in a given run is the one presented actively by the system to the user. Story management runs at the service of this story. In characterbased narrative on the other hand, there can be as many stories being created concurrently as there are characters. Story becomes situated in the experience of each character and is internalised in a process we describe as storification [3] rather than being visible from a single standpoint as 'an' external artefact.

Given the difficulty of authoring detailed interlocking multiple stories, never mind interactive variations on them all, this has an impact both on the authoring process - which becomes more declarative - and the subsequent management process. There is an interesting parallel here with Live Action Role Playing games (LARP) in which the aim is for every one of multiple participants to have a satisfying experience, in some cases of a specifically narrative type, which is jointly constructed by their activity and shaped in a limited number of ways by the GM.

The second difference follows from the first. With multiple stories, rather than checking adherence to any specific story, management focuses on character engagement in the overall process. This requires metrics allowing engagement to be detected, as with the human GM [17]. Facilitation and shaping rather than direction is then needed.

These differences limit the usefulness of some existing work on story management as envisaged here. For example, one view of story management sees it as plan repair, consistent with a plotcentred approach in which user interactivity is a source of plan failure. In [25], re-planning at execution time finds alternative ways of achieving goals that user interaction may have failed. From the perspective of emergent narrative, a problem with this approach is that it easily produces out-of-role behaviour by characters that exist only in the service of the plot.

Treating characters as semi-autonomous makes story management akin to film direction [4, 19], where again the director's vision of the single story that will be presented to the audience is central. Here the issue becomes one of the levels of direction, still in the service of 'the' story. For example, if characters have a multi-level architecture as is common in robotics, one may intervene at the level of behaviours or at the level of plans. In [4], three levels of direction were identified: overruling the character's internal architecture completely; selecting actions that are not contradicted by the character's behaviour selection system; modify the way in which an action selected by the behaviour system is carried out.

Taking this approach to its conclusion [20] argues against character autonomy on the basis that characters making local decisions cannot be responsible for the global story-state. This approach recasts story management as selection of beats from a comprehensive database, which can be thought in the robotics context already mentioned as universal plans. Interactivity is dealt with by aiming for a complete coverage of the possible beats in relation to user interaction. This approach rests on an argument that high-level management concerned with episodes or whole scenes is not enough, and once management intervenes inside scenes, character autonomy is an obstacle.

However in the emergent narrative approach, global storystate only has to have certain characteristics, rather than being composed of definite sequences. This is much more like the approach of Weyhrauch [31] which Mateas and Stern [20] are specifically arguing against. Weyyhrauch [31] formulated a story as a set of plot-points, similar in nature to the GM way-points discussed below. The drama manager monitored the state of the world and looked for triggers defining a plot-point transition. It treated this in a way analogous to a move in chess, with its own repertoire of changes in the world as possible countermoves. It evaluated all possible future story-states added to the current story-state on some metric and made what it assessed to be its 'best' move. This seems a potentially expensive process and raises the question of what would make a suitable evaluation metric. It is however an approach on which the work of section 4 has drawn.

\section{The role of a Game Master (GM)}

The role of the GM is a recurrent theme in interactive storytelling research $[9,24,17,7,32,27,29]$ and the activity of the GM is often described at a high-level so as to illustrate successful real-time interactive storytelling management. The basic principles of GM operation have been the subject of investigation within hobbyist communities [e.g. 9, 32] and have been described in RPG game products and debated in hobbyist conventions [e.g. 5]. However the cognitive processes and lowlevel mechanics involved in both single-player and multi-player RPGs are complex and have not been studied in any detail. The best work to date has been carried out in the hobbyist environment [e.g. 16, 14, 32, 21]. While some recent empirical work has been carried out on the functionality and communication of GMs and players in Pen-and-Paper RPGs as well as multi-player digital RPGs [27, 29, 30, 28 (in review)], further work is required in order to formalize the role of the GM in RPGs, and more importantly to identify the GM functions and mechanisms that can be transferred to and implemented in an interactive drama context.

The inspiration taken from earlier work in robotics in the current study relates GM-controlled processes in run-time to hierarchical planning where the planning activity consists of guiding characters through story "way-points" which have some similarity to the plot points discussed by Weyhrauch [31]. A 
"way-point", in this particular context, could be regarded as a particular state of the interactive narrative, with way points differentiated from each other by a set of changes in both the characters and the environment with an overall dramatic purpose.

A GM's planning activity can be viewed as constraining the range of behaviors used by characters to act in a story environment so as to ensure desired way points are achieved. With respect to the EN theory, the GM would not directly control the behaviors of Non-Player Characters (NPCs) but initiate goals and configure autonomous NPCs so as to shape (rather than control) the unfolding of a story. From a technical perspective, NPC behaviors could be run autonomously via AI software, independently from each other and information and intentions could be exchanged between GM and NPCs via an agent framework.

In this section, this idea is taken a step further by focusing on three relevant features of game-mastering: The distribution of authorial control in RPGs, the abstraction level(s) at which the GM operates when managing interactive stories, and the flow or run-time planning of game-mastering. While the following is admittedly brief, it should give some basic insight into both the complexity of the GM's role in multi-player RPGs, and the need for a proper analysis of means by which the GMs conceptualize and manage digital interactive storytelling environments.

\subsection{Principles of authorial control in RPGs}

Game Masters in Pen-and-Paper RPGs may have a range of responsibilities, including the provision of information about the fictional game world and developing the game storyline in collaboration with the players. The players formulate responses to changes in the fictional game world state based on the input and descriptions provided by the GM, and construct their private mental models of the events taking place [27]. The GM is thus not only in charge of the story and of communicating the state of the environmental content, but also of the flow of the game process.

A key concept of RPGs is authorial control, and how this is distributed between the players and the $\mathrm{GM}(\mathrm{s})$ of a game session [32]. Understanding how authorial control can be distributed in these games is the first step towards studying GM planning techniques and processes.

The way that authorial control is divided in Pen-and-Paper RPGs, and the variations in this feature during game play, impacts on the available space for maneuvering and management that the GM has, and therefore directly upon the formation of the collaborative story. The GM's ability to make decisions about the game world and story as well as the entities involved (for example, Non-Player Characters - NPCs), depends on the agreement between the game participants as to how authorial control is to be shared. In essence, a Pen-and-Paper RPG could operate without a GM. The greater the authorial control given to the participating players, the more adaptable and flexible a GM has to make their game story management.

However the ability of a player to affect the fictional game world in a RPG need not be directly proportional to their actual level of control over the game story, giving an illusion of greater control than is in fact available. For example, if a player destroys an object for which the GM had planned a specific use in the game story, the GM may subsequently introduce an alternative with the same story-based functionality.

The relationship between the perceived and actual authorial control of players is important to story management. In extremes such as those represented by most digital RPGs (e.g. Oblivion,
Neverwinter Nights, Fallout), players are usually limited to basic interactions with game world objects and very simple conversations with NPCs [13].

In this style of story management, the players have little or no effective impact on the game story. The GM maintains a high level of story control and defines the level of variance permitted to the players. The GM may manipulate the event flow to eliminate outcomes of player actions that are undesired and actual player control of the story may be limited to a few simple branches. However once an increased level of actual authorial control is given to the players, their decisions have more impact on the collaborative narrative [32]; and the demands on the GM to maintain flexibility as well as narrative coherence increase.

\subsection{Abstraction level(s)}

The distribution of authorial control and the relationship between actual and illusory control of the interactive story are significant high-level principles in relation to the storytelling process in Penand-Paper RPGs with a GM. However the underlying cognitive processes relating to the management and development of the interactive story are complex. The GM will typically have a series of story-based goals: a deep understanding of how these goals are managed across different levels of abstraction is critical for the successful representation of GM activity. This is a difficult area given the wide range of actions covered by GMs over different types of RPGs.

A GM manages goals at different levels of abstraction and both the overall flow of the story and the nature and effects of interaction with the players will normally be considered at multiple levels. There is a high-level, with very little detail, at which the major interactions and events are considered for inputs and outputs. This level consists of identifying what is necessary for major events to occur, in moving between way points, and what would be their repercussions on the succeeding parts of the story. Story parts are considered at lower levels, depending on how important they are or have become as a result of interaction.

While the number of abstraction levels probably varies from story-to-story or GM-to-GM, the following example gives a fair representation of the possible different levels of abstraction involved in the GM's role (Table 1).

\begin{tabular}{|l|l|}
\hline High level & The GM intends the player to gain information \\
\hline $\begin{array}{l}\text { Abstraction } \\
\text { level 2 }\end{array}$ & GM decides the bearer of information \\
\hline $\begin{array}{l}\text { Abstraction } \\
\text { level 3 }\end{array}$ & $\begin{array}{l}\text { GM decides the details of interaction between } \\
\text { players and bearer }\end{array}$ \\
\hline Lower-level & $\begin{array}{l}\text { When does it happen? What is said by NPCs? } \\
\text { What is the furniture, location? }\end{array}$ \\
\hline
\end{tabular}

Table 1. Example abstraction levels

These levels of abstraction are interdependent given that highlevel decisions determine actions/decision at the lower levels and low-level actions often also affect high-level planning and the course of a story. From a planning perspective, a common idea in hierarchical approaches such as the one shown in Table 1, where lower-levels are expansions of higher-levels is that for the higherlevel goals to succeed, the lower-level ones must too. In the case of this example, at planning time, the high-level "Pass 
information" goal, would be expanded into sets of lower-level goals so as to satisfy the high-level goal in a number of different ways. Generating alternative sets of low-level goals in order to achieve success for a high-level goal allows the planning system to cover different users or situations and gives more planning flexibility. It also means that failure to achieve pre-conditions at the low-level does not always imply failure at the high-level as alternative ways to achieve a goal can be triggered via re-planning at the lower level. This flexibility is central to the task of the GM.

However, it is important that the GM conceptualization proposed reflects the possibility that some failures at low-levels can affect high-level goals. This could be implemented by dynamically managing abstraction levels via the generation and elimination of goals during the running of a game session. Empirical data could be used to identify how variation in interaction causes deviation from pre-planned plot lines, and where these operate with respect to a hierarchical plan.

\subsection{The flow of Game-Mastering}

Tychsen [27] described RPGs as cyclic, feedback-dependant information systems in an attempt to model the overall process of information flow. While the model is useful for describing the game process at run-time, it was not concerned with capturing the narrative fluidity of Pen-and-Paper RPGs, which similarly cannot be represented within a rigid hierarchical expansion.

Fluidity is often the human attribute that is the hardest to capture in any field, and it is likely that representing the narrative flow that a GM facilitates will require the separation of RPG activity into game management and game execution. These impact each other and their interplay should be represented in detail in both the GM and character models. This is similar to AI planning where, in this particular case, plan generation would be part of the GM roe with plan execution represented by the character's activity.

On the other hand, it would seem that GMs manage the unfolding game story on many different levels and aiming to represent it as a single process within the set of GM activities would tend towards oversimplification. While player interaction at run-time is a separate concern, the interplay between the story management and player-GM interaction [27] can be subtle and can be thought of within the framework of continuous planning (Table 2) [22].

\begin{tabular}{|l|l|}
\hline 1 & $\begin{array}{l}\text { Monitor execution and mark last dispatched action } \\
\text { succeeded or failed }\end{array}$ \\
\hline 2 & $\begin{array}{l}\text { Re-organize goals: create new ones, abandon ones that } \\
\text { cannot be met, decide relative importance }\end{array}$ \\
\hline 3 & $\begin{array}{l}\text { Plan next cycle: extend one of partial plans in the current } \\
\text { set of active plans }\end{array}$ \\
\hline 4 & $\begin{array}{l}\text { Execute an action: dispatch a primitive action in one of } \\
\text { the plans if there is one }\end{array}$ \\
\hline
\end{tabular}

Table 2. Continuous planning processes.

Where GM activity differs from the classic continuous planning approach is that many of the actions in its plans are not directly dispatched. The GM instead relies on the players to execute these autonomously. The GM must therefore maintain models of characters' activities both actual and probable to project specific actions to be carried out in the context of the story. While this is not the traditional style of planning, some work has considered it [Laird]. The complexity of predicting character actions depends on how detailed a story needs to be and how densely the GM maintains commitments to specific events happening and specific states of the story-world.

The issue of commitment is another divergence from the usual approach to continuous planning. It seems probable that GMs maintain variable amounts of commitment to actions in their plans, with the way-points already mentioned representing islands of high commitment, and other sections of plan being much more open to modification by player interaction.

\section{Two implementations}

While the work discussed in the previous section is still conceptual, two pieces of research work have implemented ideas related to the GM concept introduced in this paper. These consist of the FearNot! Story Facilitator [10] and the Double Appraisal Story Facilitator [18]. These implementations are deeply rooted in the cognitive and affective modelling and strongly related to research in both $\mathrm{AI}$ and the interactive storytelling domains. They are both oriented towards a character-based representation of narratives and their conception has been heavily influenced by RPG and GM practices.

\subsection{The FearNot! Story Facilitator}

The story facilitator is an agent whose task is to sequence episodes within an emergent narrative structure [17] in the FearNot! software [23]. The aim of the application is to address anti-bullying strategies via the use of empathic synthetic characters that create virtual drama scenes through their autonomous interaction. In terms of story generation, FearNot! differs from more conventional approaches as stories emerges from the interactions between agents and users, thus generating emergent narrative. The story creation approach in FearNot! shares similarities with the GM activity in the sense that in RPGs, stories emerge from the interactions between players and GM too.

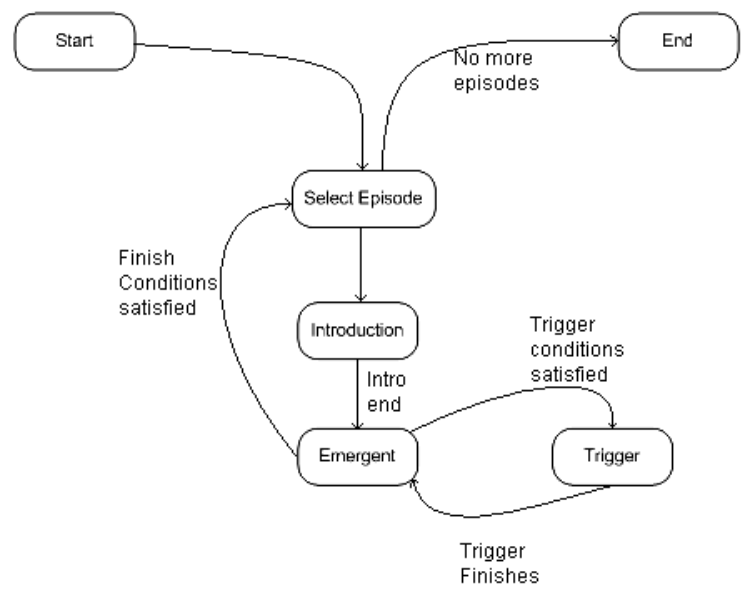

\section{Figure 1. FearNot! Story Facilitator state machine}

The story facilitator, implemented as an agent within a multiagent system, is responsible for managing the unfolding of a story. It receives and monitors, through the FAtiMA [8] agent architecture, all the messages exchanged between agents and the agent framework as well as all the messages generated within the 
framework back to the agents. This allows the story facilitator to make decisions based on the actions the agents intend to execute. Thus, the story facilitator has the ability to know everything that happens in the virtual environment. This is coupled with the ability to perform narrative actions that affect the environment. These actions are primarily dedicated to story management and are limited in its present version to the selection and set up of episodes, including decisions concerning stage, characters, action repertoires and episode types. This approach is related at a highlevel to some of the activities of the GM. The system behavior when supervised by the Story Facilitator can be represented as a state machine, as shown in Figure 1.

Episodes have been given the attributes seen in Table 3 . When the story facilitator is in the episode selection state of Figure 1, one episode is selected from the group of episodes that have their preconditions satisfied. After the selection of the episode, its introduction (set up) is executed within the graphical environment. This introduction is composed of narrative actions and typically includes actions such as narrating an introductory text or inserting the characters on the set.

\begin{tabular}{|c|c|}
\hline Attribute & Description \\
\hline Name & A unique name for the episode \\
\hline Set & $\begin{array}{l}\text { The set is the location in the virtual environment } \\
\text { where the events of this episode will take place. }\end{array}$ \\
\hline Characters & $\begin{array}{l}\text { The characters of the story, defined through a set } \\
\text { of properties like their name, position on the set, } \\
\text { etc }\end{array}$ \\
\hline Preconditions & $\begin{array}{l}\text { A set of conditions that specify when is the } \\
\text { episode eligible for selection. }\end{array}$ \\
\hline Goals & $\begin{array}{l}\text { Character goals that are communicated to the } \\
\text { agents in this particular episode. }\end{array}$ \\
\hline Triggers & $\begin{array}{l}\text { A condition that when satisfied will cause the } \\
\text { execution of a set of narrative actions. }\end{array}$ \\
\hline $\begin{array}{l}\text { Finish } \\
\text { Conditions }\end{array}$ & $\begin{array}{l}\text { A set of conditions similar to the preconditions } \\
\text { that when satisfied indicate that the episode is } \\
\text { finished. }\end{array}$ \\
\hline Introduction & A set of narrative actions introducing the episode \\
\hline
\end{tabular}

Table 3 Attributes of an episode

After the introduction, the next state in Figure 1 is the emergent state where the goals of the characters dictate the unfolding of the story. When in this state the story facilitator tests for any trigger eligible to be fired. If there are triggers that can be fired, the trigger that has the higher priority as defined by the author is selected for execution. The trigger state is similar to the introduction state, in that when the system is in this state, a set of narrative actions is executed in order, and when they finish, the story facilitator goes back to the emergent state.

An episode ends when its"ending conditions" are satisfied and the system goes back to the episode selection state. If there are no more episodes, the story finishes.

In the case of FearNot!, there is no user interaction within episodes, however given that characters are acting autonomously, the story facilitator still has to cope with unpredictable interactions. The role it plays can be thought of as a simplified version of Weyhrauch [31] in which triggers allow it to execute a sequence of narrative actions. These triggers are explicitly authored and in this version of the story facilitator there is no evaluation function allowing it to vary the sequence executed as the result of a specific trigger. It currently holds no larger-scale story-related structure, and the concept of plot-points or waypoints are implicitly rather than explicitly represented in the design of specific triggers.

\subsection{The double appraisal Story Facilitator}

The double appraisal story facilitator is also based on the FAtiMA agent architecture. It aims at managing an emergent narrative in a distributed manner so that the story facilitator itself is supported by characters that take the dramatic impact of their actions into account. This approach exploits the hypothesis that the emotional impact (EI) of an action is related to its dramatic impact, and may be used as a substitute for dramatic value. It allows the characters to conjointly assume in a distributive manner the dramatic weight of an unfolding story without relying on a plot structure. Characters do not select actions solely based on their motivations and goals, but also on the emotional impact of this action either on themselves or on other characters in the scenario.

The concept develops a novel agent action-selection mechanism featuring a double appraisal cycle, as opposed to the single appraisal system featured in other cognitive appraisal-based agent architectures. The agent appraises events as in any conventional appraisal-based system and generates emotions within the agent mind, but also runs another appraisal cycle in parallel. In this second appraisal cycle, the set of possible actions from the first appraisal cycle is assessed as if they were events according to the potential emotional impact of each action. Rather than selecting the action with the highest value for the character state after appraisal, the one with the highest emotional impact is chosen.

The story facilitator is now given the same double appraisal apparatus as characters, with the significant difference that the actions it evaluates are actually narrative actions. As before, these include initial goal sets for characters, the distribution of objects in the environment, and also the outcome of physical actions which are otherwise indeterminate. Examples of this last set of actions include whether a character falls if it is pushed and whether a character is killed, wounded or the bullet misses if it is shot. The story facilitator will consider the emotional impact of such an event upon the characters before it selects it.

One of the main features of the GM in managing stories in RPGs is the ability to assess the players' interests and apply corrective measures in order to shape the story experience of players via involvement and engagement. While this is a particularly difficult ability to model, the double appraisal features a modified action-selection mechanism in which the agent makes decisions with respect to the dramatic impact of actions (i.e. Emotional impact). The consideration of emotional impact (EI) and emotion intensity within the double appraisal concept could be regarded as a means to shape a character's experience. It offers a novel evaluation function for the story facilitator different from that of Weyhrauch [31]. It embodies the philosophy that the detail of what happens in an interactive story is less important than its impact on the participating characters, an approach consistent with the flexibility of GMs discussed above.

The double appraisal process is implemented by examining the emotions associated with specific character goals, and both the EI an action would have if directed towards the character and towards all the agents present in a scenario. The agent assesses how a potential action would be perceived by others in order to make a choice between competing potential actions. Since goals are evaluated in FAtiMA through actions, this modification also 
impacts the goal management of the agent. In order not to affect the actual emotional state of the agent, this re-appraisal cycle is executed in parallel to the "appraisal-coping" cycle seen in Figure 2 and takes place within a second instance of the agent's mind that is not connected with the agent's running emotional state. This process aims to select the action that would have the highest overall emotional impact on any character present within the scenario. It considers the impact of actions on each character and picks the one that scores the highest value for some character in the scene. The process of re-appraisal in the agent mind is shown at the foot of Figure 2.

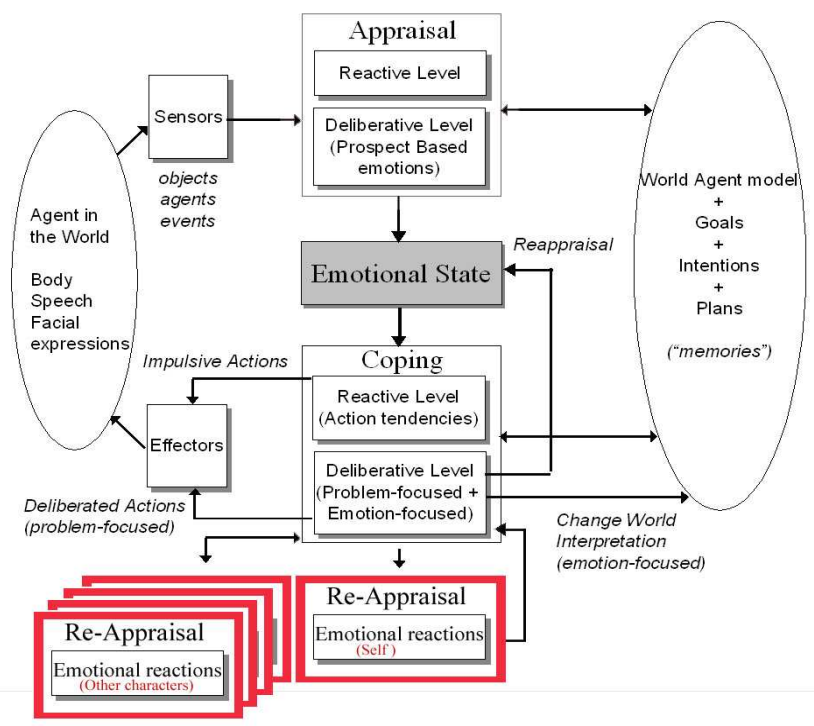

Figure 2. The FAtiMA agent architecture.

The EI consists of the difference in intensity for each emotion represented in a character before and after an action is considered for selection. The emotion intensity is a variable that fluctuate depending on the agent's personality and current state of mind, the actions already carried out in a scenario and the time elapsed since an emotion has been generated. Thus for an action such as hitting another agent, an agent would assess the EI based on how it or others would react emotionally to being hit by another agent. The double appraisal reappraises a set of valid and eligible elements selected by the first appraisal cycle with regard to the potential EI if the action or emotion was directed towards itself and all of the other agents present in a scenario. An intention is re-appraised based on the plan to achieve it. Here the action reappraised as an imagined event is the one that satisfies the relevant goal via its post-condition definition.

\section{Conclusions and future work}

This paper has discussed the specific issues relating to the management of an emergent narrative system, Given that there is no longer a unique, pre-determined plot, the methods applied in other story managers, designed to defend such a plot against the variability introduced by interactive freedom, seem inappropriate. This is far from saying that no shaping of the emergent narrative is required for the user to have an interesting and engaging narrative experience. The role of the GM in pen-and-paper RPG and in LARP has been investigated because these are seen as genres in which narrative structure and interactive freedom are often successfully reconciled. As section 3 indicates, this role is a complex one and modelling it is still in its early stages, though hierarchical planning with varying degrees of commitment and way-points seems a promising modelling approach.

The two story facilitator implementations discussed in section 4 share with GMs an ability to allow the characters to generate narrative through their interaction. The first embodies the idea of way-points or plot-points in the concept of triggers for narrative actions, which are a small subset of those a GM might employ. The second, double appraisal story facilitator takes emotional impact as a surrogate for dramatic intensity and evaluates its possible interventions in relation to their EI. GMs also take account of the engagement of players in negotiating their component of the shared authorial control referred to above.

Neither story facilitator could be said as yet to model in any substantial way the complexity of GMs. This gives plenty of scope for further work in modelling GMs in greater depth and using such a model as a guide to more complex and functional implementations. Two directions seem initially promising on the implementation side

The first of these is to equip the story facilitator with a continuous hierarchical planner. This would differ from the approach of Riedl et al [25] in that plans would have a hypothetical character, especially at lower levels of abstraction, where they would be used to monitor character activity in relation to sections of high commitment, or way-points, in the plan. The GM might be equipped with a specific set of repair mechanisms that would form the core of its actual action repertoire. The rest of its action repertoire would be composed of actions to be invoked in specific situations as is the case for GMs who must for example decide the outcome of many actions that impact the story-world and the players. EI would be one factor used to evaluate the choice of action, but other factors such as degree of commitment would need to be added to this.

A second direction lies in the refinement of the double appraisal mechanism discussed. It currently reappraises both reactive actions and plans in isolation from what has gone before. The mechanism could however be extended to take emotional trajectories into account through maintaining an EI history. There seems scope here for allowing the story facilitator to apply ideas such a dramatic climax in its choice of actions. Combining this with the first idea of maintaining a hierarchical plan with variable commitment would allow narrative shaping a little more in the flexible style of the GM.

In conclusion, the concept of emergent narrative should not be seen as one in which characters are thrown together in the hope that narrative experience will emerge, RPGs in both pen-andpaper and LARP variants indicate that reconciling the creative powers of interaction and the structural imperatives of the author are feasible provided plot is seen as a guide to what actually happens rather than a strait-jacket to be imposed upon participants. The more sophisticated relationships between planning and execution developed in robotics form an interesting source of ideas also. The RPG genre seems a much more appropriate source of ideas for interactive narrative systems than film, with its extremely strong authorial control and focus on presenting a single story to a spectating audience. We expect that empirically-based models of the GM will be indispensable to applying new ideas to interactive narrative systems. 


\section{ACKNOWLEDGMENTS}

The work on FearNot! discussed was partially supported by European Community (EC) under the Information Society Technology (IST) RTD programme in the project VICTEC contract IST-2001-33310-victec. and is currently funded by the eCIRCUS project IST-4-027656-STP. The authors are solely responsible for the content of this publication. It does not represent the opinion of the European Community, and the European Community is not responsible for any use that might be made of data appearing therein.

\section{REFERENCES}

[1] Aylett, R.S; Coddington, A.M; Barnes, D.P. \& GhaneaHercock, R.A (1997) "What does a planner need to know about execution?" in: Recent advances in AI planning, eds S.Steel \& R.Alami, pp26-38,Springer, 1997

[2] Aylett, R and Louchart, S. (2003) Towards a narrative theory of VR. Special issue on storytelling. Virtual Reality Journal Volume 7.(2003). pp $2-9$.

[3] Aylett, R.S, and Louchart, S. (2007) Being there: Participants and Spectators in Interactive Narrative. Proceedings ICVS 2007, to appear

[4] Blumberg, B. and Galyean, T. 1995. Multi-level Direction of Autonomous Creatures for Real-Time Virtual Environments. In Proceedings of SIGGRAPH 95.

[5] Bøckman, P., Hutchison, R. (eds.): Collected papers for Knutepunkt 2005 - the 9th annual Nordic Conference on larp. Knutepunkt, Oslo (2005)

[6] Brooks, R. (1986) A Robust Layered Control System for a Mobile Robot, IEEE Journal of Robotics and Automation RA2(1) pp14-23

[7] Combs, N.: The Intelligence in the MMOG: From scripts to stories to directorial AI., Other players conference, Copenhagen, Denmark (2004)

[8] Dias, J and Paiva (2005), A.Feeling and Reasoning: a Computational Model.12th Portuguese Conference on Artificial Intelligence, EPIA05. 2005.

[9] Edwards, R. (2001): GNS and Other Matters of Role-playing Theory. Adept Press http://www.indie-rpgs.com/articles/1/ accessed 04.09.07

[10] Figueiredo,R., Dias,J., Paiva, A., Aylett, R., Louchart, S. (2006) Shaping Emergent Narratives for a Pedagogical Application, NILE - Narrative and Interactive Learning Environments, Aug. 2006 , pp. 27-36

[11] Fikes, R.E; Hart, P.E. \& Nilsson, N.J. (1972) Learning and Executing Generalised Robot PlansArtificial Intelligence, 3.

[12] Gat, E. (1992) Integrating Planning and Reacting in a Heterogeneous Asynchronous Architecture for Controlling RealWorld Mobile Robots. Proceedings, 10th National Conference on Artificial Intelligence, AAAI-92, pp809-815

[13] Hallford, N., Hallford, J. (2001): Swords \& Circuitry: A Designer's Guide to Computer Role Playing Games. Prima Tech, Roseville, CA. 544

[14] Henry, L. Group Narration: Power, Information, and Play in Role Playing Games. Darkshire.net, 2003.
[15] Laird, J.QuakeBots - An exploration into computer games and computer generated forces. Eighth Conference on Computer Generated Forces and Behavior Representation.2000.

[16] Logas, H. The Taoist Storyteller. In Skotos Pleasures of the Flesh Series, 2004.

[17] Louchart, S and Aylett, R (2003) Solving the narrative paradox in VEs - lessons from RPGs In: Intelligent Virtual Agents, $4^{\text {th }}$ International Workshop IVA2003 eds T. Rist, R.Aylett, D.Ballin, LNAI 2792 Springer 2003 pp244-248

[18] Louchart, S. and Aylett, R. (2007) From synthetic characters to virtual actors. Proceedings, $3^{\text {rd }}$ Artificial Intelligence and Interactive Digital Entertainment International Conference (AIIDE 2007), AAAI Press, Marina del Ray 2007 Stanford USA. pp 88 - 91

[19] Magerko, B; Laird, J.E; Assanie, M; Kerfoot, A \&Stokes, D (2004) AI Characters and Directors for Interactive Computer Games. Procedings. of the $16^{\text {th }}$ National Innovative Applications of AI Conference, 2004

[20] Mateas, M \& Stern, A (2000) Towards Integrating Plot and Character for Interactive Drama - Working notes of the Social Intelligent Agents: The Human in the loop, AAAI Fall Symposia 2000

[21] Mäkelä, E., Koistinen, S., Siukola, M. and Turunen, S. The Process Model of Role Playing. In Dissecting Larp, Proceedings of the 2005 Knutepunkt conference, 2005.

[22] Myers, K.L. (1998) Towards a Framework for Continuous Planning and Execution, In Proceedings of the AAAI Fall Symposium on Distributed Continual Planning, 1998

[23] Paiva, A., Dias, J., Sobral, D., Aylett, R., Sobreperez, P., Woods, S., et al. (2004, July 19-23). Caring for Agents and Agents that Care: Building Empathic Relations with Synthetic Agents. Paper presented at AAMAS 2004, New York.

[24] Peinado, F. \& Gervás, P. (2004): Transferring Game Mastering Laws to Interactive Digital Storytelling. In: S. Göbel et al. (eds.): Proceedings of TIDSE 2004, 48-54.

[25] Riedl, M \& R. Michael Young (2004) An intent-driven planner for multi-agent story generation. Proceedings of the Third International Joint Conference on (2004), the Autonomous Agents and Multiagent Systems, 2004. AAMAS 2004. pp. 186193.

[26] Tychsen, A., Hitchens, M., Brolund, T. and Kavakli, M. (2005) "The Game Master". In Proceedings of the Interactive Entertainment Conference (Sydney, Australia, 2005), pp. 215222.

[27] Tychsen, A. (2006): Role Playing Games - Analysis across Two Media Platforms. In ACM Proceedings of the 3rd Australasian Conference on Interactive Entertainment (Perth, Australia), pp. 75-82.

[28] Tychsen, A. (in review): Tales for the Many - Game Process and Game Masters in Multi-Player Role Playing Games. In review for Games \& Culture.

[29] Tychsen, A.; Heide-Smith J.; Hitchens M. \& Tosca, S. P. (2006): Communication in Multi-Player Role Playing Games The Effect of Medium. In Proceedings of TIDSE 2006 (Darmstadt, Germany), pp. 277-288. 
[30] Tychsen, A.; Newman, K.; Brolund, T. \& Hitchens, M.

(2007): Cross-format analysis of the gaming experience in multiplayer role playing games. In press for Proceedings of the Digital Games Research Association Conference 2007 (Tokyo, Japan).

[31] Weyhrauch, P. 1997. Guiding Interactive Drama. Ph.D. thesis, Tech report CMU-CS-97-109, Carnegie Mellon University.

[32] Young, J. M. (2005): Theory 101: The Impossible Thing Before Breakfast. Places to Go, People to Be, 27.

http://ptgptb.org/0027/theory101-02.html Accessed 04.09.07

[33] Zilberstein, S (1995) The utility of planning. ACM SIGART Bulletin vol6, n1 (January 1995) pp 42 - 47. 